\title{
Mycotic innominate artery pseudoaneurysm presenting as an embolic stroke
}

\author{
Sunjay Kaushal, MD, PhD, Jay G. Shake, MD, and David D. Yuh, MD, Baltimore, Md
}

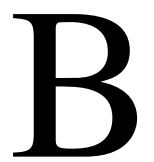

acterial, or mycotic, pseudoaneurysms of the aortic arch or its major branch vessels are rare. Aortic pseudoaneurysms have been reported as infectious complications of poststernotomy mediastinitis or endovascular aortic stent placement. ${ }^{1,2}$ We describe the unusual presentation, diagnosis, and management of a mycotic innominate artery pseudoaneurysm.

\section{Clinical Summary}

A 62-year-old man presenting with hoarseness was diagnosed with a squamous epithelial cell tumor of the soft palate. He underwent soft palate resection with adjuvant localized radiation therapy. After he was free of recurrence during the ensuing 5 years, osteoradionecrosis of the medial left clavicle and a corresponding methicillin-resistant Staphylococcus aureus (MRSA) infection involving the sternoclavicular joint developed. This was successfully treated with intravenous antibiotics, surgical debridement, and coverage with a left pectoralis muscle rotation flap.

Four months after being treated for this infection, the patient had an embolic cerebrovascular accident localized to the right middle cerebral artery territory, as determined by magnetic resonance imaging, and this manifested as a left-sided hemiparesis. Incidentally, magnetic resonance imaging also revealed a $3.6 \times 3.4-\mathrm{cm}$ saccular pseudoaneurysm with a narrow $5-\mathrm{mm}$ neck emanating from the anterior base of the innominate artery (Figure 1) and extensive inflammatory soft tissue in the anterior mediastinum extending from the left distal clavicle to the innominate, subclavian, and common carotid arteries.

The results of blood cultures were positive for MRSA, and intravenous vancomycin was initiated. Because the results of additional diagnostic studies, including a carotid Duplex ultrasound and cardiac echocardiogram, were unremarkable, the most plausible source of this embolic stroke was ascribed to the pseudoaneurysm. Because of the perceived risk of further embolic stroke and risk of rupture, pseudoaneurysm resection was recommended.

\footnotetext{
From the Division of Cardiac Surgery, Johns Hopkins Hospital, Baltimore, Md.

Received for publication Aug 8, 2004; revisions received Aug 13, 2004; accepted for publication Aug 18, 2004.

Address for reprints: David D. Yuh, MD, Division of Cardiac Surgery, Johns Hopkins Hospital, 600 North Wolfe St, Blalock 618, Baltimore, MD 21287-4618 (E-mail: dyuh@csurg.jhmi.jhu.edu).

J Thorac Cardiovasc Surg 2005;129:945-6

$0022-5223 / \$ 30.00$

Copyright (C) 2005 by The American Association for Thoracic Surgery

doi:10.1016/j.jtcvs.2004.08.018
}

Surgical resection was performed 2 weeks after the cerebrovascular accident and after near-complete recovery of left-sided motor function. At the time of the operation, the innominate

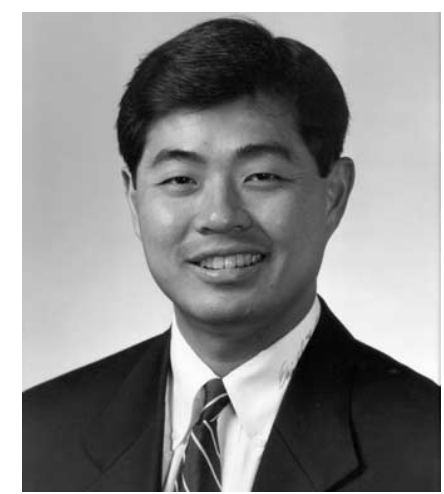

Dr Yuh artery pseudoaneurysm was identified through a standard median sternotomy approach (Figure 2). During the dissection, moderate inflammatory tissues were encountered in the vicinity of the aortic arch in continuity with the left sternoclavicular region, although no gross purulence was noted. Cardiopulmonary bypass was instituted with aortic cannulation of the distal ascending aorta and dual-staged venous cannulation of the right atrium. With the use of deep hypothermic circulatory arrest, the pseudoaneurysm was dissected circumferentially and opened. Substantial thrombus was removed from the saccular component of the pseudoaneurysm, revealing a narrow neck arising approximately $8 \mathrm{~mm}$ distal to the origin of the innominate artery. After the pseudoaneurysm was completely resected, the resultant $1-\mathrm{cm}$ defect at the base of the innominate artery was repaired with a patch of autologous pericardium fixed in glutaraldehyde. A 28-minute period of circulatory arrest was required. The results of intraoperative cultures and histologic analyses of the pseudoaneurysm and surrounding mediastinal tissues were negative for active infection or neoplasm. The patient was discharged to home after an unremarkable 7-day postoperative course to complete a 6-week course of MRSAspecific intravenous antibiotics.

\section{Discussion}

Mycotic pseudoaneurysms of the ascending aorta, aortic arch, or its primary branch vessels are rarely encountered and usually arise from perforation of an arterial wall, the integrity of which is compromised by direct trauma (eg, suture line, cannulation site, or penetrating weapon), an infective process, or both. Primary bacterial infection of the aortic wall leading to pseudoaneurysm formation is rarer still and is believed to be a sequela of bacterial endocarditis or endothelial trauma from an aortic jet lesion. Infection of laminar thrombus within a true aortic aneurysm might also be seeded during transient bactere$\mathrm{mia}^{2}$ Left untreated, pseudoaneurysms can rupture or constitute a source of thromboembolism. The most common bacterial pathogens include, in order of decreasing frequency, Staphylococcus aureus, Staphylococcus epidermidis, Salmonella species, Streptococcus species, and Pseudomonas species. ${ }^{3}$

Patients undergoing extensive head and neck resections and adjuvant radiotherapy are predisposed to vascular arteriopathy. Ischemic morphologic changes of the arterial wall after local irradiation are initially characterized by acute vascular endothelial 

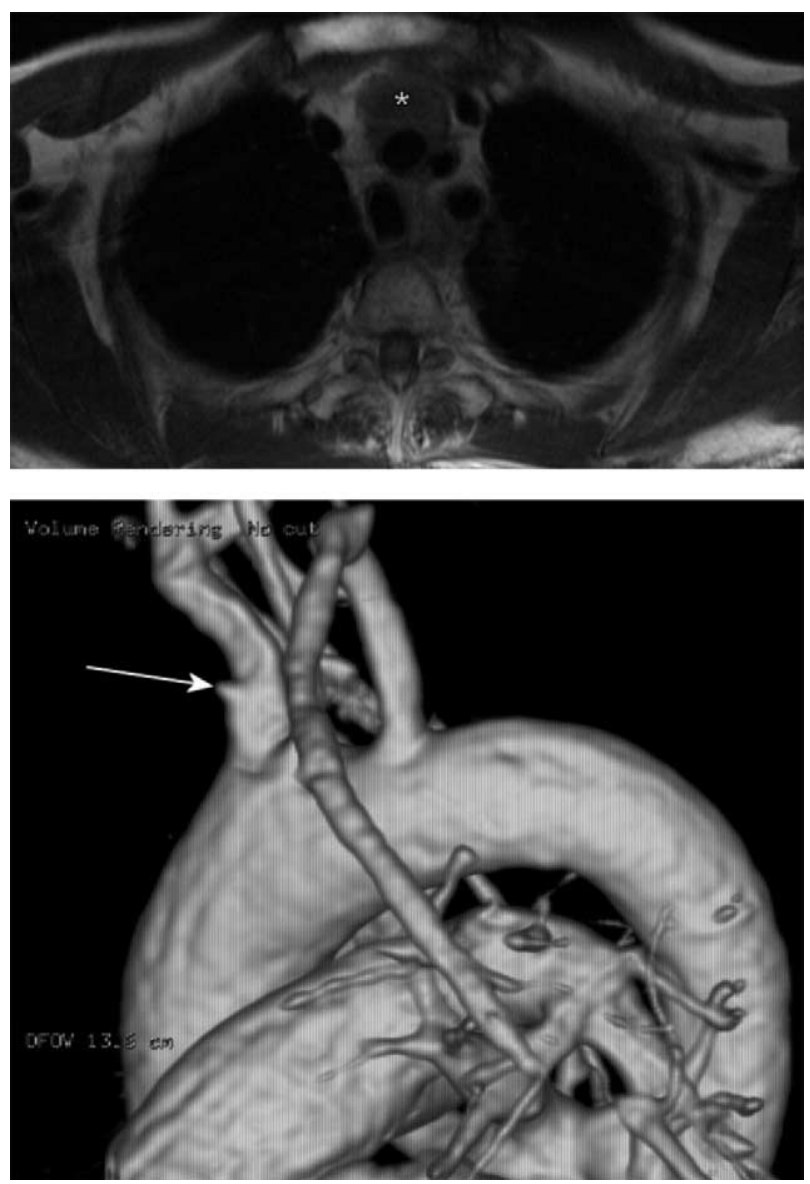

Figure 1. Magnetic resonance imaging of innominate artery pseudoaneurysm. Top panel, Cross-sectional view showing a largely thrombosed saccular pseudoaneurysm (white asterisk) with extensive inflammatory soft tissue stranding in the anterior mediastinum extending from the left distal clavicle to the innominate, subclavian, and common carotid arteries. Bottom panel, Three-dimensional reconstruction showing the narrow 5-mm pseudoaneurysm neck (white arrow) emanating from the anterior base of the innominate artery.

cell damage, followed by subacute fibrosis of the media layer with local hemorrhage and necrosis. ${ }^{4}$ These ischemic alterations, commonly attributed to occlusion of the vasa vasorum, lead to pseudoaneurysm formation ranging from 2 to 20 years after head and neck irradiation. ${ }^{5}$ Pseudoaneurysms occurring after head and neck irradiation typically arise from the external or internal carotid artery or carotid bifurcation.

In this case it is unclear whether head and neck irradiation, the resultant sternoclavicular joint infection, or the combination of these factors ultimately led to the pseudoaneurysm. Regardless of the cause, we believe that the risk of rupture and embolization warrants an aggressive surgical approach consisting of resection and repair. Reconstruction should be done with autologous tissue when possible because of the infectious nature of this rare complication.
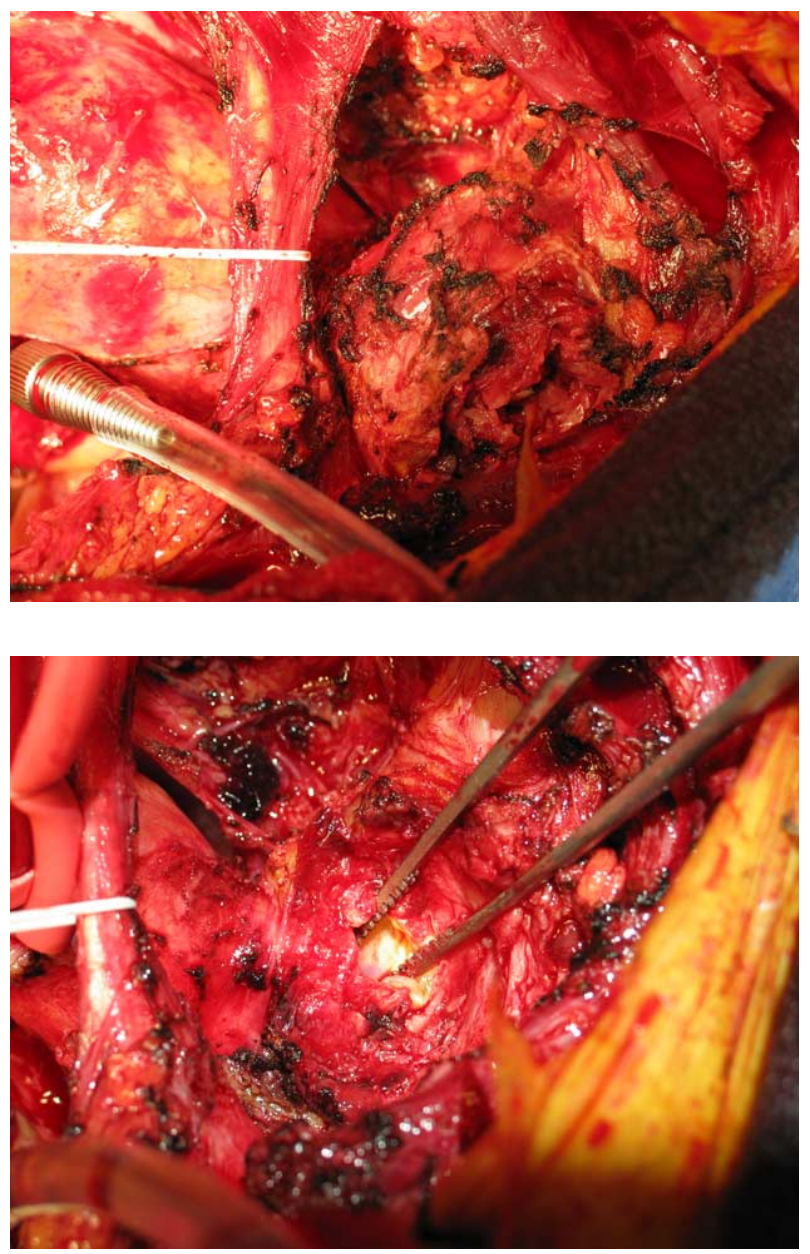

Figure 2. Intraoperative photographs showing egg-shaped innominate artery pseudoaneurysm, cephalad (to the right) of the looped innominate vein (top panel) and showing, after excision, a 1-cm defect (forceps) at the base of the innominate artery (bottom panel).

\section{References}

1. Pruitt A, Dodson TF, Najibi, S, Thourani V, Sherman A, Cloft H, et al. Mycotic aneurysm of the ascending aorta. J Vasc Surg. 2002;36(3): 625-8.

2. Anderson CA, Rizzo RJ, Cohn LH. Ascending aortic aneurysms. In: Cohn LH, Edmunds LH, editors. Cardiac surgery in the adult. 2nd ed. New York: McGraw-Hill; 2003. p. 1123-48.

3. Vrochides D, Feng WC, Singh AK. Mycotic ascending aortic pseudoaneurysm secondary to pseudomonas mediastinitis at the aortic cannulation site. Tex Heart Inst J. 2003;30:322-4.

4. Fonkalsrud EW, Sanchez M, Zerubavel R, Mahoney A. Serial changes in arterial structure following radiation therapy. Surg Gynecol Obstet. 1977; 145:395-400.

5. Ernemann U, Herrmann C, Plontke S, Schafer J, Plasswilm L, Skalej M. Pseudoaneurysm of the superior thyroid artery following radiotherapy for hypopharyngeal cancer. Ann Otol Rhinol Laryngol. 2003;112:18890. 